Proceedings of the International School and Conference on Optics and Optical Materials, ISCOM07, Belgrade, Serbia, September 3-7, 2007

\title{
Porous Silicon Avalanche LEDs and their Applications in Optoelectronics and Information Displays
}

\author{
P. Jaguiro, P. Katsuba*, S. Lazarouk and A. Smirnov \\ Belarusian State University of Informatics and Radioelectronics \\ P. Browka Street 6, 220600 Minsk, Belarus
}

The use of silicon based light emitting diodes may completely solve the problem of low compatibility of optoelectronics elements and silicon chip. At present time the most suitable kinds of Si-LEDs are monocrystal and porous silicon avalanche LEDs. They have advantages such as long operation lifetime ( $>10000$ hours), continuous spectrum, which allows to filter RGB colors, operation voltages $(<12 \mathrm{~V})$, extremely sharp voltage-current characteristic, nanosecond response time, and high high operation current densities (up to $8000 \mathrm{~A} / \mathrm{cm}^{2}$ in pulse mode). Rather low energy efficiency $(<1 \%)$ is not so significant for near to eyes (NTE) microdisplays. These advantages open a way to design a high performance and cost effective passive addressed microdisplays.

PACS numbers: 78.55.Mb, 78.60.Fi, 85.60.Jb, 85.60.Pg

\section{Introduction}

Research into the light emission from silicon has a history of more than five decades [1]. During that time silicon technology has significantly improved and correspondently the quantum efficiency of silicon LEDs was raised from $10^{6}$ to several percents [2-4]. One of the milestones on this way was the evidence of visible light emission from avalanche porous silicon (PS) reverse biased diodes [5]. At present time PS avalanche LEDs have long operation lifetime, low operation voltages $(<12 \mathrm{~V})$, extremely sharp VA-curve, nanosecond response time and high operation current densities (up to $3000 \mathrm{~A} / \mathrm{cm}^{2}$ in pulse mode). The next significant step towards to silicon LEDs is the evidence of high efficiency visible light emission on monocrystal silicon under special conditions [6] with quantum efficiency of $0.18 \%$ [7]. Background, approaches and achievements in investigation of the light emission mechanism, design of the silicon based LEDs and matrix high resolution displays will be considered in this paper.

*corresponding author; e-mail: pavel@jaguiro.com 


\section{Electroluminescence in porous and monocrystal silicon}

There are several theories explaining electroluminescence in silicon [3], but real situation is not limited by any single theory. We have used theory of optical intraband transitions of hot carriers [6]. This theory is not complete, but it is "working" and gives qualitative forecast for different microelectronic structures, which make use of porous silicon and monocrystal silicon LEDs. The difference between these kinds of silicon can be explained by changes in morphology and band structure distortion. Some examples of application of the theory will be given later in the section discussing silicon LEDs design.

The main shortcoming of the theory is its inability to explain a maximum in the emission spectrum. Some theoretical improvement can be made to overcome this problem (in particularly by taking into account indirect interband transitions for hot carriers), if relevant experimental data is collected.

\section{Electrophysical properties of Si based LEDs}

First samples of the silicon LED have an extremely low efficiency [1]. The theory can justify this if the consider the fact that the planar high voltage junctions are used. In this case avalanche conditions are achieved at low local electric field strength and corresponding hot carrier temperature is not sufficient for effective light emitting. To improve the performance it is necessary to use high doped silicon and high curved (small islands for example) junctions [6], as it is shown in Fig. 1 following the theory forecast.

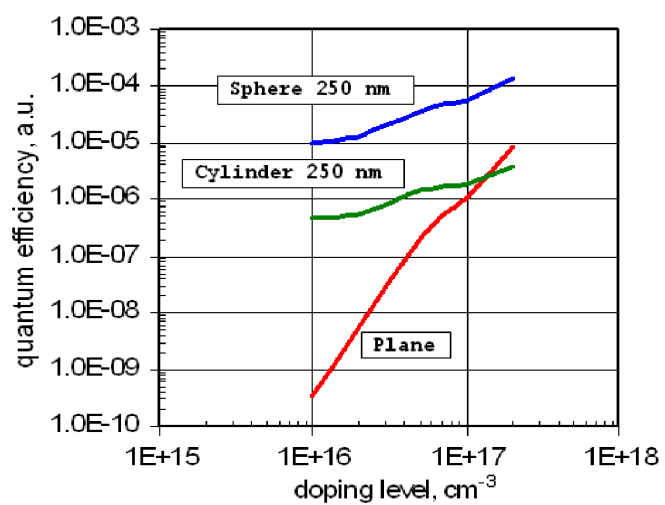

Fig. 1. Quantum yield in visible range via doping level for planar, cylinder, and spherical topologies.

Both of these changes increase the local electric field strength, however this raises the problem of tunneling breakdown. Tunneling current does not produce hot carriers and from light emission point of view it is a parasitic current which decreases efficiency. The most effective working state for silicon LEDs is in the 
mixed avalanche/tunneling breakdown regime, and to estimate the best mixed point it is necessary to use reliable microelectronic technology base. Use of porous silicon gives several advantages - the junction has a lot of microcurved areas and tunneling breakdown is depressed due to higher band gap in quantum confinements. But porous silicon dramatically changes electrical properties of LEDs, compared with original silicon, for example breakdown voltage can be occasionally raised and deviated from the most efficient point. This effect can be explained by taking into account silicon "broken filaments" — silicon parts in corralled porous structure which do not take part in through current transport. However the electrophysical behavior of porous silicon is similar to that of monocrystal silicon with higher doping level [8].

As all avalanche devices the reverse biased LEDs have extremely fast electrical time response and due rather low quantum efficiency (it means that there are high-speed alternative energy relaxation processes) optical time response is very short, too. In our measurements the total response time was as low as few nanoseconds [9].

Porous silicon structures are very chemically active due to their high surface area and so the degradation problem is crucial for LEDs. To prevent the degradation it is necessary to use specially-designed cover layers. It is appears that one of the best protection cover are an $\mathrm{Al} / \mathrm{Al}_{2} \mathrm{O}_{3}$ or $\mathrm{Al} / \mathrm{Nb}_{2} \mathrm{O}_{5} / \mathrm{Al}_{2} \mathrm{O}_{3}$ layers. These can be manufactured through a standard $\mathrm{Al}$ metallization process which includes deposition of $\mathrm{Al}$ (or Al-Nb bilayer), photolithography masking, anodizing Al through photoresist mask (or plasma etching Nb through the mask, and anodizing Al) [10]. After $1000 \mathrm{~h}$ of continuous operation no symptoms of degradation were found in the tested LEDs [5]. Probably, the lifetime is the same as it is for common semiconductor devices, especially if Al-Si alloy is used except $\mathrm{Al}$ to prevent Si dissolution in cover electrode.

Breakdown in an avalanche LED is thermally-dependent and at a critical current density the substrate heating is enough to locally decrease the ohmic resistance and concentrate the heating in the local area. Finally current filament path is formed where silicon substrate is locally melted [11]. This kind of breakdown is rather inertial and the pulsed currents can be significantly higher than the available steady state current. For example, we have achieved pulsed current densities up to $8000 \mathrm{~A} / \mathrm{cm}^{2}$ while the maximum available steady state current density for the sample was only $150 \mathrm{~A} / \mathrm{cm}^{2}[11]$.

\section{Si based optoelectronics devices}

Compared with traditional LEDs silicon avalanche LEDs have continuous emission spectra, faster response time, good stability but significantly lower energetic efficiency. It is a relatively good suit of parameters and some specific application niches are open for Si LEDs (for example, it can be used as calibrator for photomultipliers). Unfortunately, specific niches are not enough to start a com- 
mercial realization. Main advantage of the Si LEDs is its excellent compatibility with silicon microelectronic technology and corresponding integration possibilities.

The first porous silicon light emitting diode integrated with control microelectronic circuit was made by Fauchet's group; however they used direct biased porous silicon LEDs and encountered some problems with operation stability [12].

Another significant field of application is integration of silicon LEDs with photodetectors (PD) and optical waveguides. As the LEDs have very fast response time, it is possible to make effective optical inter- and intrachip connections. The first silicon optoelectronic couple was made by combining porous silicon LED, aluminum waveguide and porous silicon based photodetector $[13,14]$. The design is shown in Fig. 2.
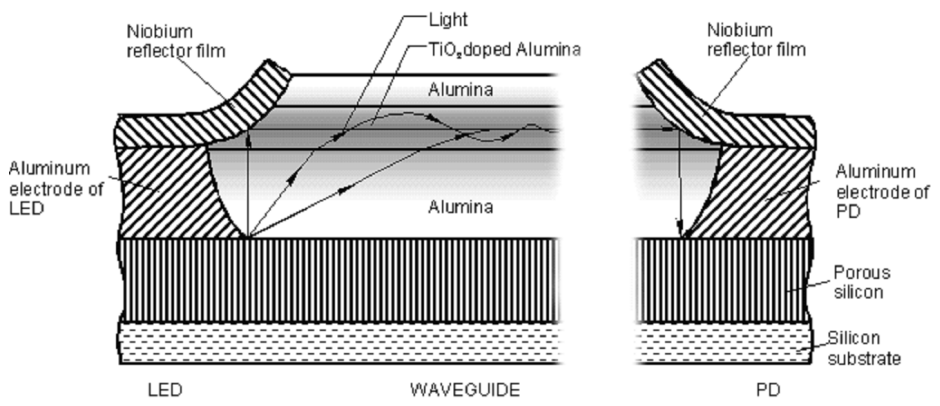

Fig. 2. Silicon optoelectronic couple.

Some attempts were made to design a high resolution display based on porous silicon LED matrix. Proposed active matrix design was rather complicated and expensive $[15,16]$ since the LEDs needed to be controlled by a current of very high density.

\section{Si based NTE microdisplays}

Until recently the research into $\mathrm{Si}$ based matrix displays was limited on academic level without any real practical yields. Main reasons of the limitation were low efficiency and small size (like a standard semiconductor chip) of the displays.

The situation has seriously changed when a market of NTE microdisplays was formed. Currently it is obvious that the emissive Si based passive addressed microdisplays, even without integration with the drivers, can be a real competitor at NTE displays arena.

When we compare the required emission energy of a traditional desktop display and the NTE microdisplay, which forms the same sized image, it appears that the difference can be up to 200-500 times. If we take into account that normal brightness of the NTE displays can be only $50 \mathrm{~cd} / \mathrm{m}^{2}$ (due to outside light being cut out) the total energy difference may be as great as 3000 times. 


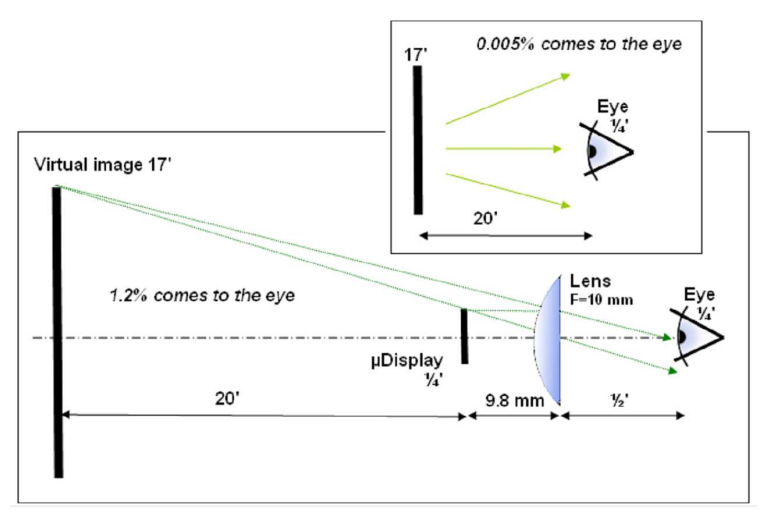

Fig. 3. Comparison of energy efficiency for standard (in the inset) and NTE displays.

If we calculate the corresponding light energy $W$ for the NTE display for the yellow spectrum region (this is real for Si LED) it should be only $0.2 \mathrm{~mW}$. For the chip power consumption $1 \mathrm{~W}$ external power efficiency should be $0.02 \%$, which is achievable for both porous silicon LEDs and for monocrystal ones.

In Fig. 3 we compare the required emission energy of a traditional desktop display and the NTE microdisplay, which forms the same sized image. Very fast response time and operation at high current densities allow to realize the deep multiplexing and to fabricate a cost effective passive addressed microdisplay. For example, if operation current density $J$ is $7000 \mathrm{~A} / \mathrm{cm}^{2}$, active area $A$ is $6 \times 6 \mathrm{~mm}$, applied voltage $V_{\mathrm{a}}$ is $8 \mathrm{~V}$ and external power efficiency $\eta$ is $0.3 \%$, it is possible to achieve multiplexing level. Currently we have made a working prototype of porous silicon based NTE display. It consists of optical system, chip with direct driving porous silicon LEDs and pulse generator. It is possible to simulate a passive addressed pixel operation at high multiplexing levels. The device shows bright, excellent visible picture even in direct sunlight condition.

\section{Conclusion}

Light emitting Si based passive addressed microdisplays, even without integration with row and column drivers, can be a real competitor at NTE arena. Unique suit of parameters allows designing cost effective high resolution displays. Porous silicon as well as monocrystal bulk silicon based LEDs can be used in these displays. Some theoretical, experimental and engineering investigations should be carried out to consolidate its place in the niche on the NTE microdisplay market.

\section{References}

[1] A.G. Chynoweth, K.G. McKay, Phys. Rev. 102, 369 (1956).

[2] X. Zhang, Electrochemistry of Silicon and Its Oxide, Kluwer/Plenum, New York 2001. 
[3] A. Halimaoui in: Properties of Porous Silicon, Ed. L.T.Canham, Short Run Press, London 1997, p. 18.

[4] B. Geloz, N. Koshida, J. Appl. Phys. 88, 4319 (2000).

[5] S. Lazarouk, P. Jaguiro, S. Katsouba, G. Masini, S. La Monica, G. Maiello, A. Ferrari, Appl. Phys. Lett. 68, 1648 (1996).

[6] P. Jaguiro, A. Ferrari, S. Lazarouk, Proc. Electrochem. Soc., PV 97-29, 194 (1998).

[7] J. Zhao, A. Wang, T. Truke, M. Green, MRS Proc. 744, M4.7 (2002).

[8] P. Jaguiro, S. Lazarouk, L. Pavesi, A. Smirnov, in: Proc. 10th Int. SID Symp., Minsk 2001, Ed. A. Smirnov, Society for Information Display, Raubichi 2001, p. 112.

[9] S. Lazarouk, P. Jaguiro, S. Katsouba, A. Prohorenko, Proc. Electrochem. Soc., PV 7, 456 (1997).

[10] S. Lazarouk, P. Jaguiro, S. Katsouba, Advanced Metallization and Interconnect Systems for ULSI, Applications, Ed. R. Cheung, Materials Research Society ,San Diego 1997, p. 11.

[11] S. La Monica, M. Balucani, S. Lazarouk, G. Maiello, G. Masini, P. Jaguiro, A. Ferrari, Solid State Phenomena 54, 21 (1997).

[12] K. Hirschman, L. Tsybeskov, S. Duttagupta, P. Fauchet, Nature 384, 338 (1996).

[13] S. Lazarouk, P. Jaguiro, V. Borisenko, Phys. Status Solidi A 165, 87 (1998).

[14] S. Lazarouk, P. Jaguiro, A. Leshok, V. Borisenko, in: Physics, Chemistry and Application of Nanostructures, World Press, Singapore 1999, p. 370.

[15] S. Lazarouk, A. Smirnov, A. Belous, S. Shvedov, in: Proc. 10th Int. SID Symp., Minsk 2001, Ed. A. Smirnov, Society for Information Display, Raubichi 2001, p. 76 .

[16] S. Lazarouk, A. Smirnov, V. Labunov, in: Proc. IDMC-03, Taiwan 2003, Ed. SID, Society for Information Display, Taipei 2003, p. 3-08. 